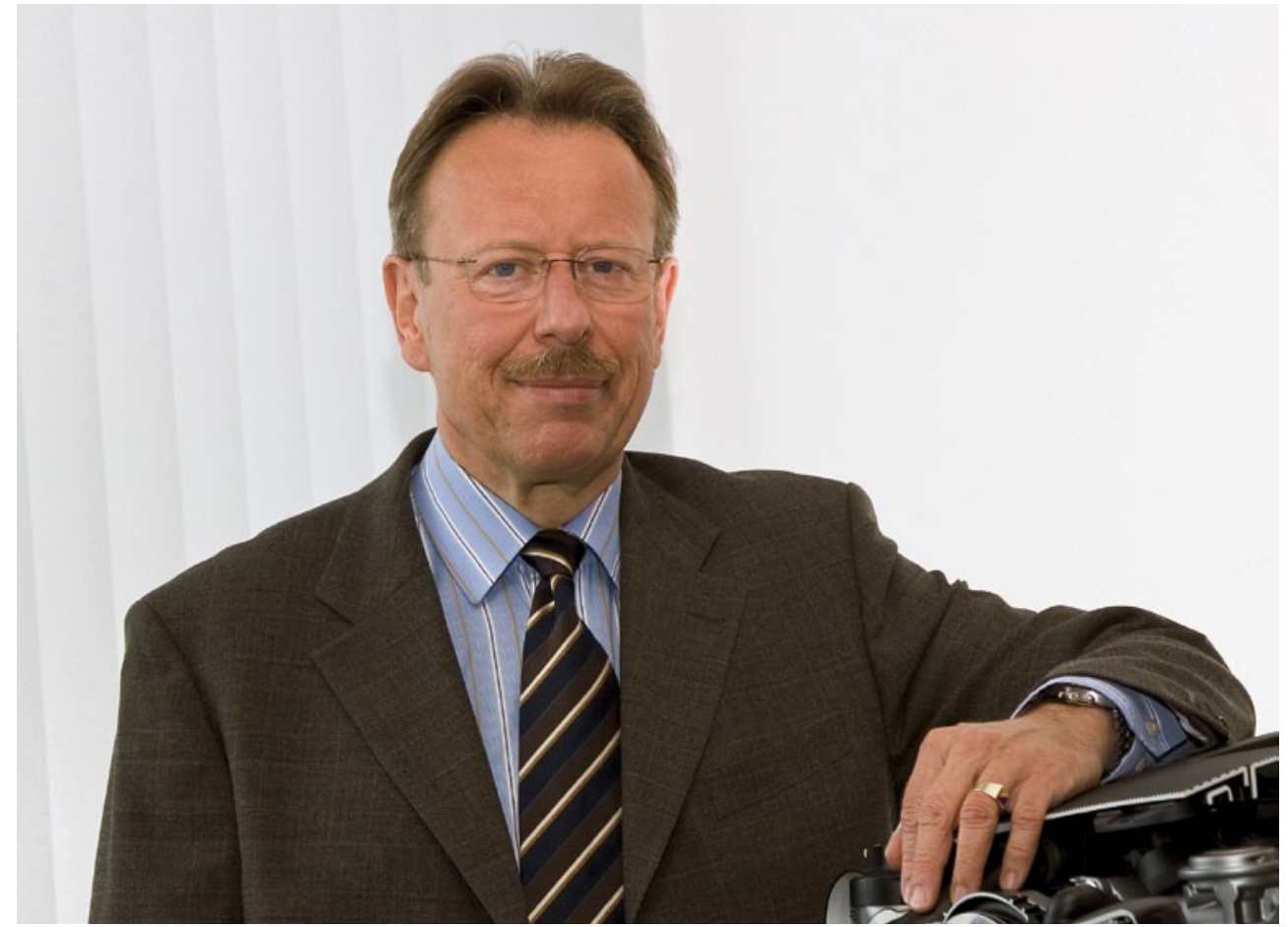

PROF. DR.-ING. LEOPOLD MIKULIC Vice President Cooperation Coordination Renault/Nissan, Daimler AG

\title{
KOOPERATION UNTER EINEM GUTEN STERN
}

Die unauffällige, aber doch effiziente Zusammenarbeit der Komponenten eines Fahrzeugantriebs miteinander, aber auch mit dem Gesamtfahrzeug, stellt heute und in Zukunft die wesentliche Grundlage für Kundenzufriedenheit und das Erreichen technischer und produktspezifischer Zielsetzungen dar. Eine vergleichbare Form der Zusammenarbeit - gleichbedeutend mit dem Begriff „Kooperation“ - beispielsweise zwischen Unternehmen ist aus unserem internen wie externen beruflichen und unternehmerischen Umfeld nicht mehr wegzudenken. Mit den stets kürzer werdenden Produkt- und Innovationszyklen und gleichzeitig gestiegenen Anforderungen an Ressourcen und Know-how ist die Zusammenarbeit mit Partnern ein wesentlicher Erfolgsfaktor insbesondere für Automobilunternehmen geworden. Damit können Produkte schneller auf den Markt gebracht werden, gemeinsam bei Investitionen Einsparungen realisiert werden oder Skaleneffekte durch den gemeinsamen Bezug von Komponenten erzielt werden. Aber auch hier muss - wie beispielsweise bei den Antriebskomponenten in einem Fahrzeug - das Zusammenspiel aufeinander abgestimmt werden. Dabei trägt jede Komponente - und im Falle der Kooperation jeder Mitarbeiter - Entscheidendes dazu bei, wie und ob das große Zusammenspiel funktioniert und erfolgreich ist.

Mit Recht steht am Beginn einer Kooperation die Frage nach der Wahrscheinlichkeit des Erfolgs. Eine große Anzahl von Erfolgsfaktoren lässt sich an uns geläufigen technischen und wirt- schaftlichen Kenngrößen festmachen, bewerten und optimieren. Oft zeigt sich jedoch, dass diese Partnerschaften anfangs ungewohnte Anforderungen mit sich bringen. Schließlich hat jeder Mitarbeiter nicht nur seine eigenen Fachkenntnisse, sondern auch eigene Vorstellungen von seiner Arbeitsweise und jener seines Partners. Hier treffen im Arbeitsalltag sprichwörtlich die Welten der Partnerunternehmen aufeinander. Die Erfahrung zeigt, dass das Kennenlernen und Verstehen der Arbeitsweise des Partners beispielsweise in spezifischen Trainingsprogrammen entscheidend dazu beitragen kann, dieses Problem zu lösen. In einem geeignet ausgestalteten „Excellence Programm“ lernen die Firmenteams zunächst die Unternehmenskultur der Partner kennen, daran anschließend werden gemeinsam umfassende Zusammenarbeitsmodelle entwickelt. Großes Augenmerk kommt für eine erfolgreiche Zusammenarbeit nicht nur der Vermeidung unterschiedlicher Interpretationen von Begriffen zu, sondern auch der Auseinandersetzung mit den fallweise unterschiedlichen Prozessen des Partners. Die Erarbeitung eines gemeinsamen Prozessverständnisses und beispielsweise die gemeinsame Festlegung des Produktentstehungsprozesses, einschließlich der Meilensteine und deren Inhalte, ist in der Anfangsphase eines Kooperationsvorhabens unabdingbar und ein wesentlicher Erfolgsfaktor.

Mit einer erfolgreichen Kooperation verhält es sich im Grunde wie bei einem erfolgreichen Automobil - die optimale Zusammenarbeit optimaler Komponenten ist der Schlüssel zum Erfolg. 